\title{
FROTTEMENT INTÉRIEUR ET MICRODÉFORMATION DANS LES TRANSFORMATIONS MARTENSITIQUES
}

\author{
J. F. DELORME *, R. SCHMID, M. ROBIN et P. GOBIN \\ Laboratoire de Métallurgie Physique et de Physique des Matériaux, I. N. S. A., Lyon
}

\begin{abstract}
Résumé. - Le comportement de trois alliages $\mathrm{Fe}-\mathrm{Ni}$, Co-Ni et $\mathrm{Fe}-\mathrm{Cr}-\mathrm{Ni}$ a été étudié pendant leurs transformations martensitiques en les soumettant soit à une contrainte statique de torsion très inférieure à la limite élastique conventionnelle ce qui entraîne une microdéformation plastique, soit à une contrainte sinusoïdale du même ordre de grandeur ce qui permet la mesure du frottement intérieur.

Nous avons montré notamment que dans les trois alliages, pour une transformation donnée :

- La microdéformation plastique est proportionnelle à la contrainte statique appliquée.

- Le frottement intérieur passe par un maximum dans le domaine de température correspondant à la transformation.

Une étude phénoménologique nous a permis de prévoir l'influence de divers paramètres expérimentaux tels que l'amplitude et la fréquence de mesure, la vitesse de transformation, sur la valeur du frottement intérieur et d'établir un lien formel entre ce dernier et les mesures de microdéformation. Ces prévisions sont comparées aux résultats expérimentaux et discutées.

Abstract. - The martensitic transformation of three alloys, $\mathrm{Fe}-\mathrm{Ni}$, $\mathrm{Co}-\mathrm{Ni}, \mathrm{Fe}-\mathrm{Cr}-\mathrm{Ni}$ have been studied. These alloys were submitted to two types of stresses:

- A static torsional one lower than their usual yield stress. This involves a plastic microstrain during the transformation.

- A cyclic stress due to internal friction measurements.

It has been shown for these alloys that :

- The plastic microstrain is proportionnal to the applied static stress.

- There is a maximum of internal friction in the transformation temperature range.

A phenomenological analysis allows us to forecast the influence of different experimental parameters such as amplitude, frequency, transformation rate, on the anelastic properties of the materials.

Deductions of this model are compared with experimental results.
\end{abstract}

I. Introduction. - En 1937, Wassermann [1] introduisit le premier le terme de «Plasticité de transformation » pour décrire la résistance à l'écoulement particulièrement faible observée pendant le changement de phase $\gamma \rightarrow \alpha$ d'un alliage Fe-Ni.

Un phénomène semblable avait déjà été observé par Sauveur [2] dans le fer et depuis lors, des études de déformation plastique pendant les transformations ont été faites sur les aciers, le titane et ses alliages, le zirconium, le laiton $\beta$, le cobalt, l'uranium ; une revue bibliographique des principaux résultats est donnée dans [3]. En général, pour des contraintes suffisamment faibles, la déformation plastique totale pour un cycle de température dans le domaine de transformation directe et inverse est une fonction linéaire de la contrainte appliquée. Le phénomène se produit aussi bien lors des changements de phase avec diffusion que sans diffusion (martensitiques).

La plupart des essais ont été conduits au dilatomètre sous contrainte de traction ou de compression, ou lors d'essais de traction conventionnels, mais Kot et

\footnotetext{
* Cet article regroupe en partie le travail d'une Thèse qui sera soutenue par J. F. Delorme devant l'Université Claude Bernard de Lyon.
}

al. [3] ainsi que Garber et Datsko [4] ont également travaillé en torsion.

Il apparaît également que le frottement intérieur passe par un maximum dans le domaine de température correspondant à une transformation allotropique. C'est notamment le cas pour des alliages fer-nickel $[5,6,7]$, le cobalt $[8,9,10]$, le titane [11], le zirconium et le beryllium [12], le fer [4]. La vitesse de variation de la température dans le domaine de transformation semble jouer un rôle important. Pour des alliages fer-nickel en particulier, Scheil et Muller [7] ont montré que la capacité d'amortissement liée à une transformation allotropique présentait une nature très différente de celle observée dans un processus de relaxation classique.

Nous nous sommes donc attachés à étudier pendant leurs transformations le comportement de divers alliages soumis, soit à une contrainte statique de torsion très inférieure à la limite élastique conventionnelle ce qui entraîne une microdéformation plastique, soit à une contrainte sinusoïdale du même ordre de grandeur ce qui permet la mesure du frottement intérieur et de la période d'oscillations. Ces méthodes doivent permettre d'aborder l'étude de l'interaction matricemartensite au cours de la transformation. 
TABleau I

\begin{tabular}{|c|c|}
\hline Composition & Transformations \\
\hline $\mathrm{Fe}-30 \% \mathrm{Ni}$ & cubique faces centrées $\rightarrow$ cubique centre \\
\hline $\mathrm{Co}-30 \% \mathrm{Ni}$ & $\begin{array}{ll}\text { cubique faces centrées } & \rightarrow \text { hexagonal compact } \\
\text { hexagonal compact } & \rightarrow \text { cubique faces centrées }\end{array}$ \\
\hline $\mathrm{Fe}-17,7 \% \mathrm{Cr}-7,4 \% \mathrm{Ni}$ & cubique faces centrées $\Varangle \begin{array}{l}\text { hexagonal compact } \\
\text { cubique centré }\end{array}$ \\
\hline
\end{tabular}

Nous avons choisi pour cette étude trois alliages $\mathrm{Fe}-\mathrm{Ni}, \mathrm{Co}-\mathrm{Ni}$ et $\mathrm{Fe}-\mathrm{Cr}-\mathrm{Ni}$ pour lesquels nous indiquons dans le tableau I la composition et la nature des transformations subies.

Le caractère martensitique de la transformation $\gamma \rightarrow \alpha$ des alliages fer-nickel a été mis en évidence par Jones et Pumphrey [13]. Pour des teneurs en nickel comprises entre 29 et $32 \%$, la martensite apparaît en plaquettes de forme lenticulaire présentant une microstructure finement maclée de part et d'autre d'un plan médian. La formation très rapide de quantités importantes de martensite est une caractéristique de ces alliages : il semble qu'elle soit due à une sorte de réaction en chaîne, la formation d'une plaquette entraînant celle de très nombreuses autres plaquettes dans le même grain et dans les grains voisins. Ce phénomène est appelé « burst » et se traduit parfois par un déclic audible. Dans l'alliage étudié $M_{S}$ est voisin de $-70^{\circ} \mathrm{C}$ et la transformation est presque totale à l'air liquide. $\mathrm{La}$ transformation inverse au chauffage commence vers $400^{\circ} \mathrm{C}$.

Entre 0 et $30 \%$ de $\mathrm{Ni}$, les alliages $\mathrm{Co}-\mathrm{Ni}$ peuvent exister sous deux formes allotropiques, l'une c. f. c. stable à haute température, l'autre h. c., stable à basse température. Une telle transformation a notamment été étudiée par cinémicrographie [14]. Lorsque la teneur en nickel croît, la stabilité de la phase cubique est favorisée. La phase cubique devient stable dès l'ambiante au-delà de $30 \% \mathrm{Ni}$, et l'on constate alors une croissance de l'énergie de défaut d'empilement avec la teneur en Nickel [15]. Dans l'alliage dont nous disposons, la transformation au refroidissement commence dès l'ambiante; la transformation inverse au chauffage commence vers $100^{\circ} \mathrm{C}$ et se termine vers $200^{\circ} \mathrm{C}$

Enfin, il est maintenant bien établi que les aciers inoxydables de type 18/8 subissent une destabilisation par transformation martensitique lors d'un refroidissement à basse température conduisant à l'apparition de 2 phases, l'un $\varepsilon$, hexagonale compacte, l'autre $\alpha$ cubique centrée. Ces transformations martensitiques peuvent se produire de façon isotherme et anisotherme $[16,17]$. La transformation $\gamma \rightarrow \alpha$ semble prépondérante [18] ; il semble établi que sa vitesse passe par un maximum pour une température intermédiaire. Les transformations inverses de ces deux phases se produisent en général au-dessus de l'ambiante. Dans l'alliage étudié, $M_{S}$ est voisin de $-50^{\circ} \mathrm{C}$, la vitesse de transformation $\gamma \rightarrow \alpha$ est maximum vers $-115^{\circ} \mathrm{C}$ et la transformation directe qui débute par refroidissement de la température ambiante à celle de l'air liquide reprend faiblement au réchauffage ; toutefois, après un tel cycle, la quantité transformée reste faible $(\simeq 15 \%)$.

Nous avons donc choisi d'étudier 2 transitions cristallographiques simples et une troisième plus complexe qui présente des éléments appartenant à chacune des deux précédentes. D'autre part, les trois familles d'alliages choisies présentent l'avantage d'avoir été déjà abondamment étudiées sous l'angle de la transformation martensitique, notamment par dilatométrie, mesures de paramètres aux $\mathrm{RX}$, microscopies optique et électronique.

La plasticité de transformation a déjà été mise en évidence par microdéformation dans ces alliages. D'autre part, des mesures d'amortissement lors de la transformation ont été faites notamment par Scheil [67] sur le fer-nickel, Postnikov [19-20], Selle [21] sur le Co, Delorme [22] sur les aciers inoxydables austénitiques.

Toutefois, ces études sont partielles et aucune tentative n'est faite pour comparer les résultats. C'est pourquoi nous avons choisi de conduire parallèlement les essais de microdéformation et de frottement intérieur sur ces trois types d'alliages.

II. Techniques expérimentales. - Les éprouvettes sont des fils de diamètre 1,8 à $2 \mathrm{~mm}$ et de longueur utile $33 \mathrm{~mm}$.

Les traitements thermiques appliqués aux éprouvettes avant les essais sont indiqués dans le tableau II.

\section{Tableau II}

$\overline{\mathrm{Fe}-30 \% \mathrm{Ni}}\left|\begin{array}{l}\text { Recuit de } 20 \mathrm{~h} \text { à } 750 \circ \mathrm{C}- \\
950^{\circ} \mathrm{C}, 1100^{\circ} \mathrm{C}\end{array}\right|$\begin{tabular}{l} 
Refroidissement à l'air \\
\hline $\begin{array}{l}\text { Recuit de } 70 \mathrm{~h} \text { à } 1100^{\circ} \mathrm{C} \\
\text { en ampoules de quartz } \\
\text { scellées }\end{array}$
\end{tabular} \mid \begin{tabular}{l} 
Refroidissement au four \\
\hline
\end{tabular}

$\mathrm{Fe}-\mathrm{Cr}-\mathrm{Ni}$ Recuit de $20 \mathrm{~mm}$ à $1050^{\circ} \mathrm{C}$ Hypertrempe à l'eau 
Après traitement les éprouvettes ont subi un maintien de 24 heures au moins à l'ambiante avant essai.

Les éprouvettes sont montées sur un pendule de torsion inversé habituellement utilisé pour la mesure $\mathrm{du}$ frottement intérieur et de la période d'oscillation.

Pour l'essai de microdéformation on fait passer un courant continu I dans les bobines du système électromagnétique de lancement du pendule : on exerce ainsi un couple de torsion constant sur l'éprouvette.

Un étalonnage préalable de ce dispositif à l'ambiante permet de vérifier que : - le lot d'éprouvettes est suffisamment homogène (dispersion inférieure à $5 \%$ ).

- Les contraintes appliquées aux échantillons sont dans le domaine élastique à la température ambiante.

- Le couplage bobines-aimants est constant dans le domaine des essais.

Un suiveur de spot permet d'enregistrer en fonction du temps la déformation $\varepsilon$ de la fibre extérieure de l'éprouvette grâce à un miroir solidaire de l'élément mobile du pendule.

III. Résultats expérimentaux. - III.1 MICRODÉFORMATION. - III.1.1 On appellera $\varepsilon_{i}$ la déformation élastique initiale des éprouvettes sous l'effet de la contrainte de torsion constante appliquée, et $\varepsilon_{p}$ la déformation plastique supplémentaire qui se produit lors de la transformation.

Lorsque les éprouvettes ne sont soumises à aucun couple $\left(\varepsilon_{i}=0\right)$ on observe une torsion $\varepsilon_{p}$ dont le sens est aléatoire. Ce phénomène semble lié à la relaxation des contraintes internes lors de la transformation martensitique [6]. On considère en première approximation que la déformation $\varepsilon_{p}$ est nulle pour $\varepsilon_{i}=0$. étant donnée sa faible amplitude.

$\mathrm{Si}$ au contraire, une contrainte élastique est appliquée $\left(\varepsilon_{i} \neq 0\right)$, on enregistre une déformation $\varepsilon_{p}$ dans le sens de la contrainte.

Nous avons porté sur les figures 1,2 et 3 , des exemples de courbes donnant l'allure des variations de $\varepsilon_{p}$ en fonction de la température pour les trois alliages.

Pour l'alliage $\mathrm{Fe}-\mathrm{Ni}$, la courbe représentée figure 1 correspond à un $\varepsilon_{i}$ de $15 \times 10^{-5}$ à $20^{\circ} \mathrm{C}$. On distingue nettement des zones de déformation brutale (A) et de déformation continue (B). Les zones A correspondent très probablement au phénomène de «burst " habituel dans ces alliages.

La figure 2 représente les résultats obtenus pour l'alliage Co-Ni. Les déformations au chauffage et au refroidissement sont notées $\varepsilon_{p 1}$ et $\varepsilon_{p 2}$; elles correspondent respectivement à la transformation h. c. $\rightarrow$ c. f. c. et à la transformation inverse; dans les deux cas, les concentrations relatives de phase transformée sont approximativement égales et voisines de $50 \%$. On constate que les valeurs $\varepsilon_{p 1}$ et $\varepsilon_{p 2}$ correspondantes sont aussi presque égales. Les vitesses maximums de transformation sont indiquées par les points d'inflexion qui se situent vers $180^{\circ} \mathrm{C}$ au chauffage et vers $-40^{\circ} \mathrm{C}$ au refroidissement.

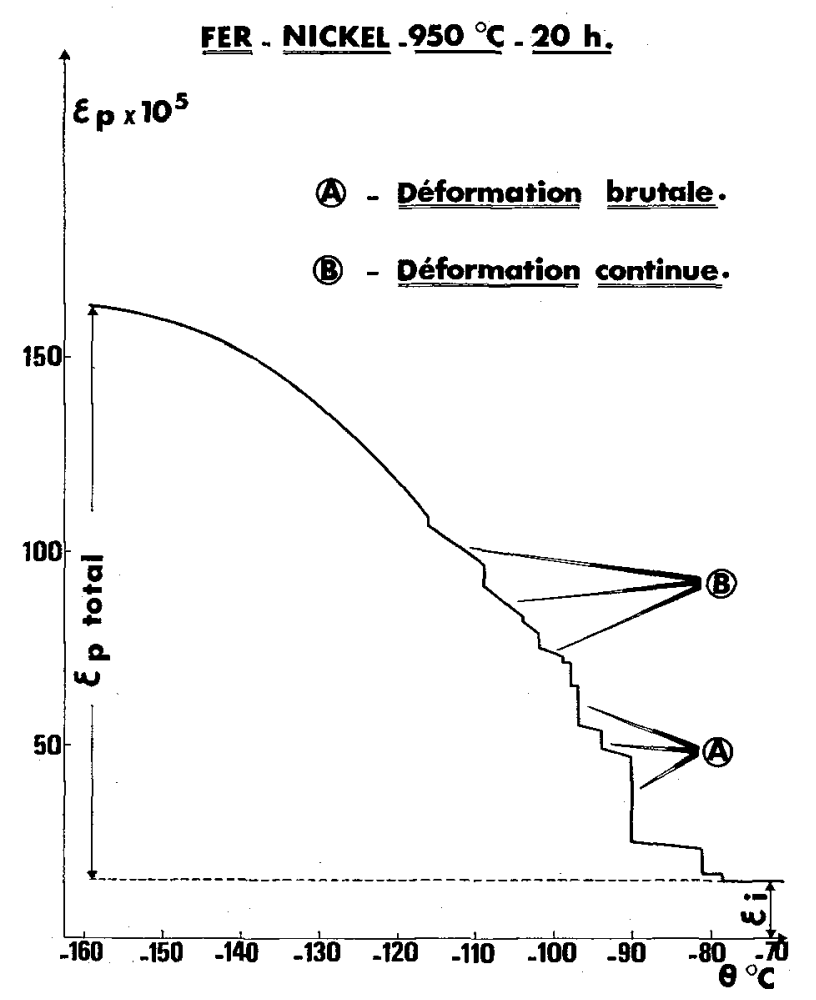

FIG. 1. - Alliage Fer-Nickel. Déformation plastique $\varepsilon_{p}$ en fonction de la température au cours d'un refroidissement. $\left(\varepsilon_{i}=15 \times 10^{-5}\right)$.

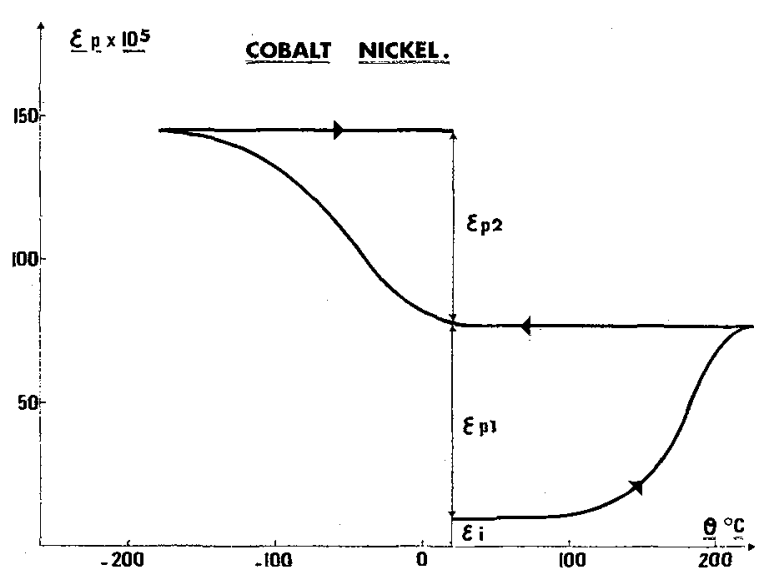

Fig. 2. - Alliage Cobalt-Nickel. Déformation plastique $\varepsilon_{p}$ en fonction de la température au cours d'un chauffage et d'un refroidissement $\left(\varepsilon_{i}=10 \times 10^{-5}\right)$.

Pour l'alliage $\mathrm{Fe}-\mathrm{Cr}-\mathrm{Ni}$, le cycle thermique comprend un refroidissement de l'ambiante à $-175^{\circ} \mathrm{C}$ suivi d'un réchauffage jusqu'à l'ambiante. Les points $A$, $B, C$ et $D$ de la figure 3 représentent la déformation obtenue après refroidissement, les points $\mathrm{A}^{\prime}, \mathrm{B}^{\prime}, \mathrm{C}^{\prime}$ et $\mathrm{D}^{\prime}$, la valeur de $\varepsilon_{p}$ pour le cycle complet, pour 4 valeurs croissantes de $\varepsilon_{i}$. On constate que $\varepsilon_{p}$ croit avec $\varepsilon_{i}$ et qu'au refroidissement $\varepsilon_{p}$ commence à croître à une température supérieure quand $\varepsilon_{i}$ est plus grand. On vérifie d'autre part que la transformation n'est pas totale au refroidissement et reprend plus faiblement 


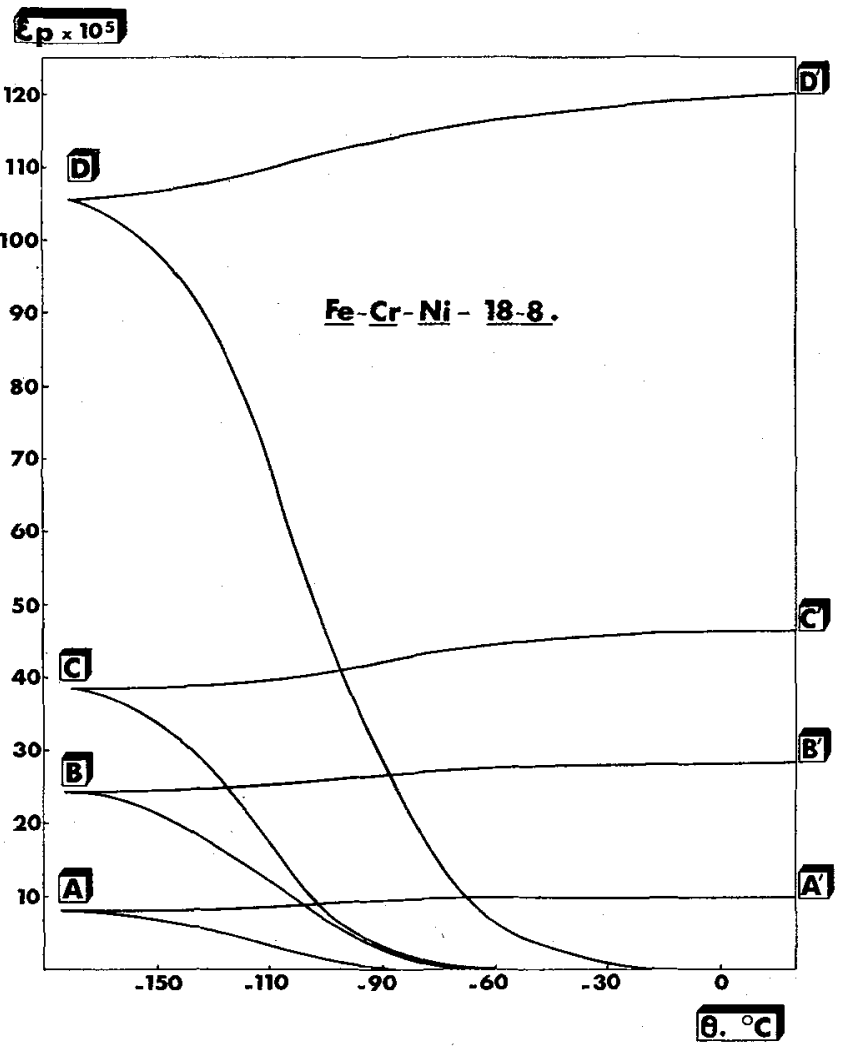

Fig. 3. - Alliage Fer-Chrome-Nickel. Déformation plastique $\varepsilon_{p}$ en fonction de la température au cours d'un refroidissement et d'un réchauffage pour 4 contraintes statiques :

$$
\begin{array}{ll}
\text { A. } \varepsilon_{i}=2 \times 10^{-5} & \text { B. } \varepsilon_{i}=4,5 \times 10^{-5} \\
\text { C. } \varepsilon_{i}=8,5 \times 10^{-5} & \text { D. } \varepsilon_{i}=17,5 \times 10^{-5}
\end{array}
$$

au réchauffage: les points d'inflexion situés sur les 2 branches de chaque courbe révèlent l'existence d'une vitesse maximum de transformation située vers $-115^{\circ} \mathrm{C}$.

On voit donc que, dans les trois cas, $\varepsilon_{p}$ varie dans le sens de la contrainte appliquée, quelque soient le type de transition cristallographique et le sens dans lequel s'effectue la transformation.

III.1.2 Nous avons étudié l'évolution de la variation de $\varepsilon_{p}$ en cours de transformation pour différentes valeurs de $\varepsilon_{i}$. Les figures 4,5 et 6 représentent les courbes $\varepsilon_{p}=\mathrm{f}\left(\varepsilon_{i}\right)$ ainsi obtenues pour les trois alliages.

Pour l'alliage $\mathrm{Fe}-\mathrm{Ni}$, les courbes $\mathrm{A}$ et $\mathrm{C}$ de la figure 4 représentent respectivement la somme des déformations plastiques brutales et la déformation plastique totale. On peut remarquer que :

- Les courbes $\mathrm{A}$ et $\mathrm{C}$ sont des droites.

- Les résultats présentent une certaine dispersion, mais la précision s'améliore lorsque la contrainte appliquée augmente.

- Pour les très faibles valeurs de $\varepsilon_{i}$ on a tracé un rectangle d'incertitude : la dispersion dans cette zone est en effet telle qu'il est impossible de tracer une courbe.

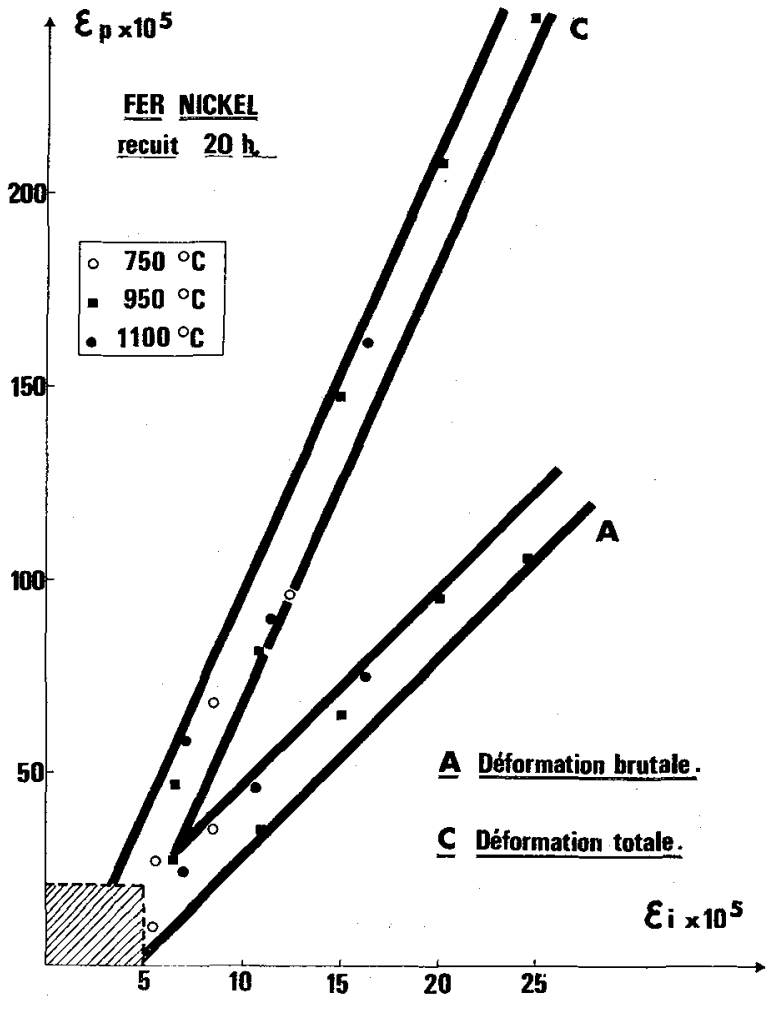

Fig. 4. - Alliage Fer-Nickel. Déformation plastique $\varepsilon_{p}$ en fonction de la déformation élastique appliquée $\varepsilon_{i}$.

$A$. Pour la déformation brutale (Burst).

$C$. Pour la déformation totale $(A+B)$.

(Influence des traitements thermiques).

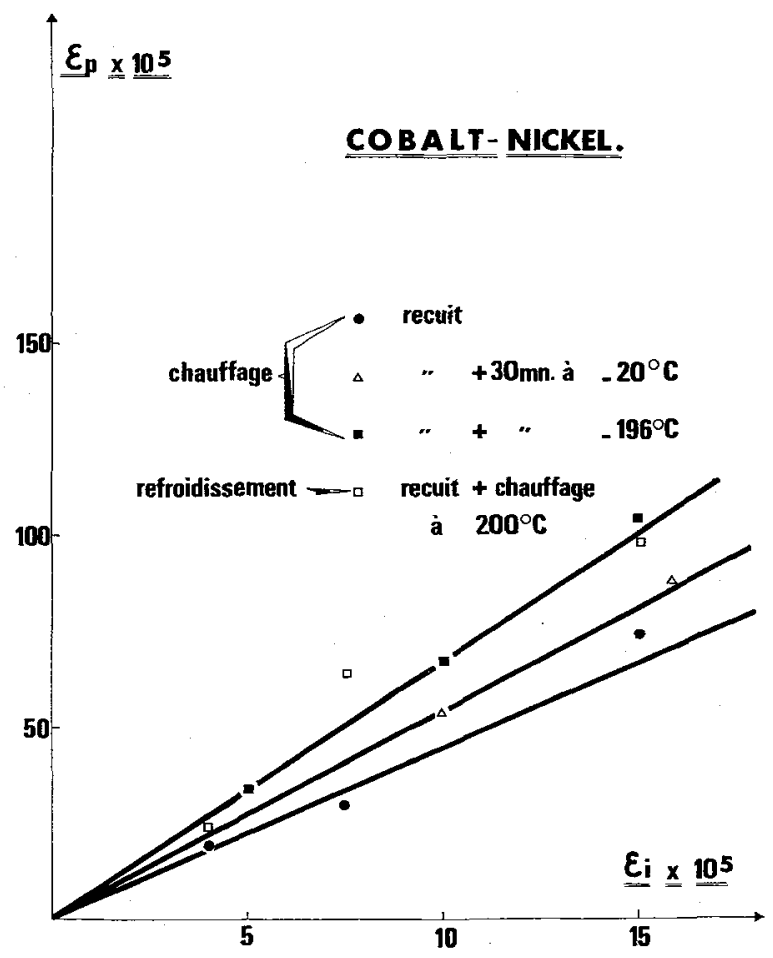

Fig. 5. - Alliage Cobalt-Nickel. Déformation plastique $\varepsilon_{p}$ en fonction de la déformation élastique appliquée $\varepsilon_{i}$; pour différentes concentrations de phase transformée. 


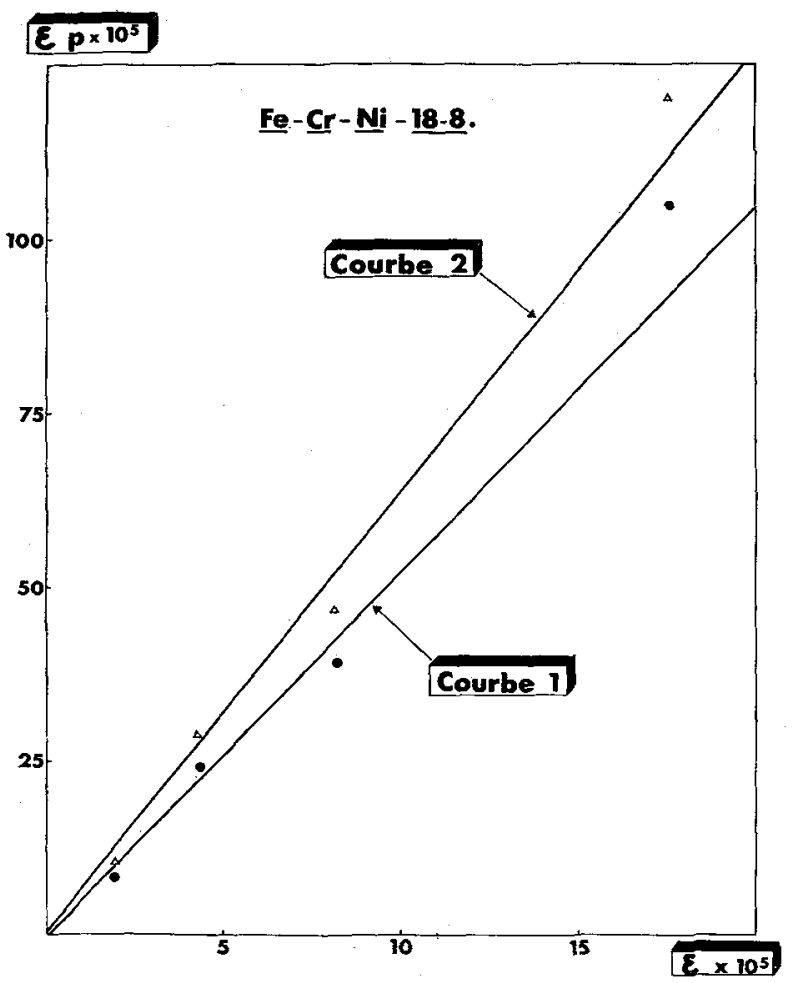

FIG. 6. - Alliage Fer-Chrome-Nickel. Déformation plastique $\varepsilon_{p}$ en fonction de la déformation élastique appliquée $\varepsilon_{i}$.

Courbe 1 . Après refroidissement à $-175^{\circ} \mathrm{C}$;

Courbe 2. Après refroidissement à $-175^{\circ} \mathrm{C}$ et réchauffage à $20^{\circ} \mathrm{C}$.

- Il ne semble pas y avoir d'effet des traitements thermiques préalables.

Pour l'alliage $\mathrm{Co}-\mathrm{Ni}$, les résultats portés sur la figure 5 correspondent à 4 cas différents :

- Chauffage à partir de l'état recuit.

- Chauffage à partir de l'état recuit et refroidi à $-20^{\circ} \mathrm{C}$.

- Chauffage à partir de l'état recuit et refroidi à $-196^{\circ} \mathrm{C}$

- Refroidissement après chauffage à $200^{\circ} \mathrm{C}$.

Les trois premiers cas sont relatifs à la transformation h. c. $\rightarrow$ c. f. c., pour des quantités initiales croissantes de phase $h$. c., la quatrième à la transformation inverse c. f. c. $\rightarrow$ h. c. On constate que les courbes obtenues sont approximativement des droites dont la pente augmente avec la quantité de phase mise en jeu lors de la transformation.

Nous avons représenté figure 6 les résultats obtenus pour l'alliage $\mathrm{Fe}-\mathrm{Cr}-\mathrm{Ni}$. Les courbes 1 et 2 correspondent respectivement aux points $A, B, C, D$ et $\mathrm{A}^{\prime}, \mathrm{B}^{\prime}, \mathrm{C}^{\prime}, \mathrm{D}^{\prime}$ de la figure 3 , donc à des quantités de phase transformée croissantes. On constate que ces deux courbes sont des droites, la pente de la droite 2 étant supérieure à celle de la droite 1 .

Nous pouvons donc dire, quel que soit l'alliage considéré, que les courbes $\varepsilon_{p}=\mathrm{f}\left(\varepsilon_{i}\right)$ sont en première approximation des droites passant par l'origine, ce qui montre qu'à la précision des mesures près et dans le domaine de variation de $\varepsilon_{i}$ considéré, $\varepsilon_{p}$ est proportionnel à $\varepsilon_{i}$. De plus, le rapport $p=\varepsilon_{p} / \varepsilon_{i}$ semble varier dans le même sens que la concentration relative $n$ de phase transformée.

III. 2 Frottement intérieur. - Nous avons effectué sur des échantillons des mêmes alliages, au cours de cycles thermiques respectifs identiques des mesures de frottement intérieur et de période d'oscillation à une fréquence de mesure voisine de $1 \mathrm{~Hz}$.

Les résultats obtenus pour la mesure du décrément logarithmique pour les trois alliages sont portés sur les figures 7, 8 et 9 en fonction de la température.

Sur la figure 7 nous avons indiqué les variations de $\delta$ avec la température pour 3 amplitudes de mesures différentes, dans le cas de l'alliage Fe-Ni. Dans l'inter-

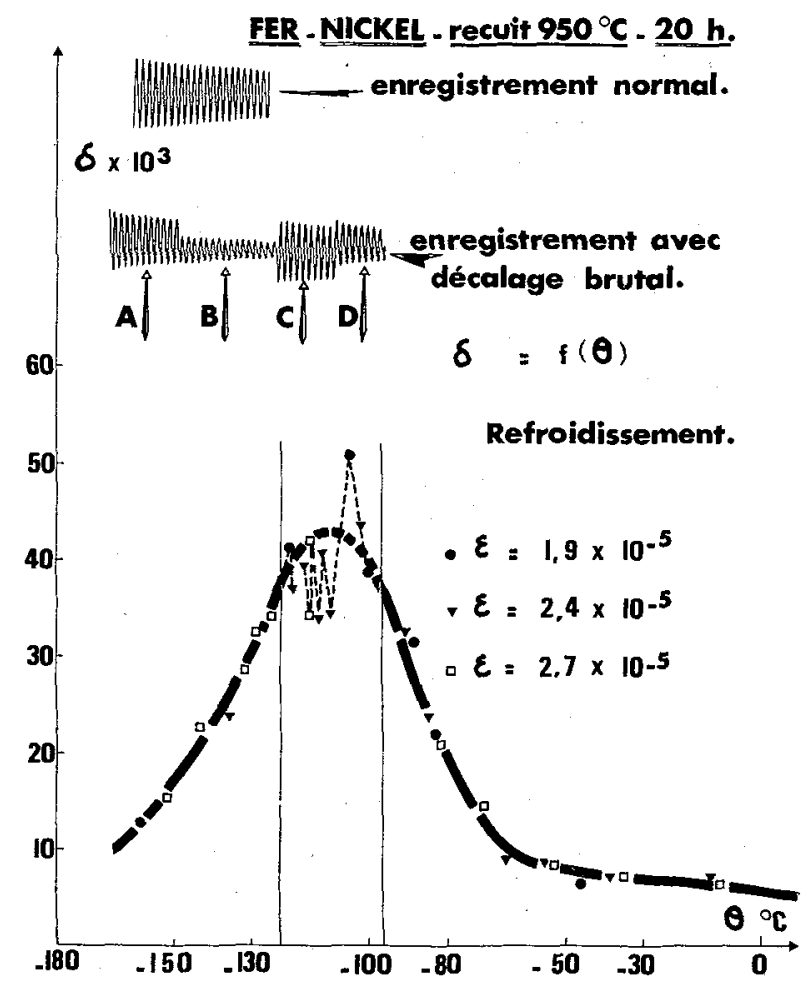

FIG. 7. - Alliage Fer-Nickel. Variation du frottement intérieur au cours du refroidissement de l'alliage. Influence de l'amplitude de la déformation.

valle compris entre $-90^{\circ} \mathrm{C}$ et $-120^{\circ} \mathrm{C}$, la courbe a été représentée en pointillés car les mesures présentent alors une dispersion considérable. On a également représenté deux exemples d'enregistrement des. oscillations du pendule, l'un normal et l'autre, qui comporte des décalages brutaux de la position d'équilibre, correspondant à l'intervalle $-90^{\circ} \mathrm{C},-120^{\circ} \mathrm{C}$ : on comprend que ces décalages rendent les mesures très délicates dans cette zone. Cependant on a vérifié,

- que le frottement intérieur passe par un maximum au cours de la transformation.

- que le frottement intérieur est indépendant de 


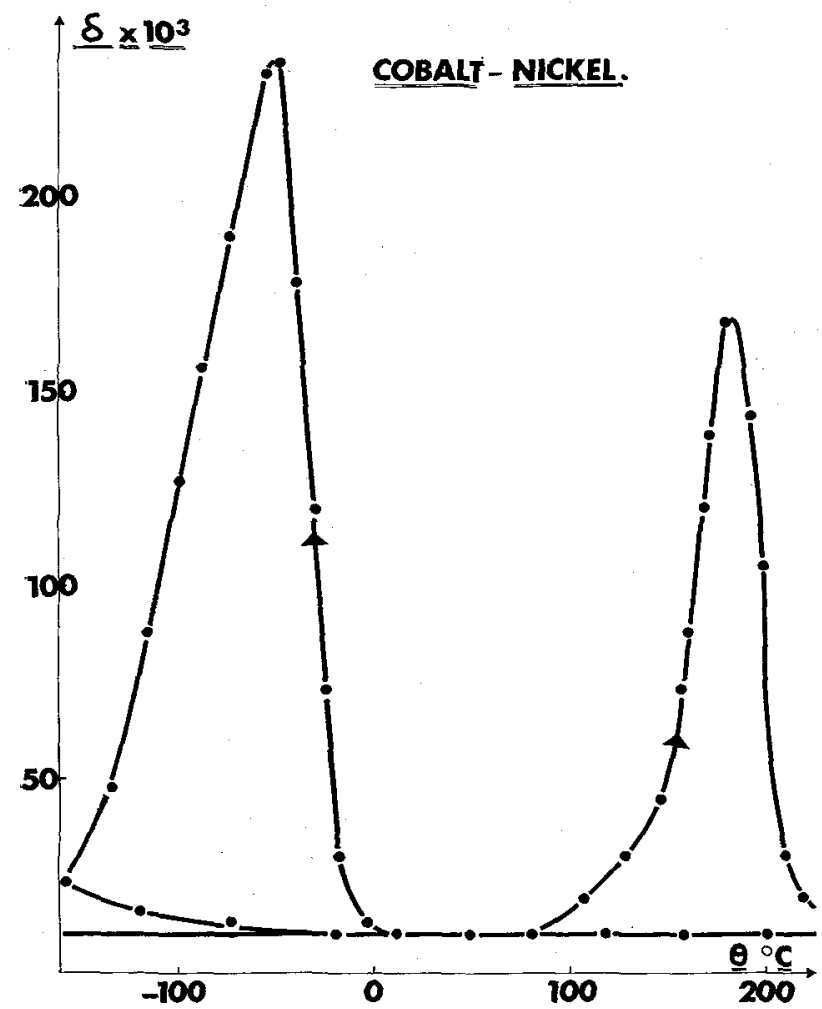

FIG. 8. - Alliage Cobalt-Nickel. Variation du frottement intérieur au cours de la transformation h.c. $\rightarrow$ c. f.c. (chauffage) et c. f. c. $\rightarrow$ h. c. (refroidissement) mesure effectuée à $\varepsilon=2 \times 10^{-5}$.

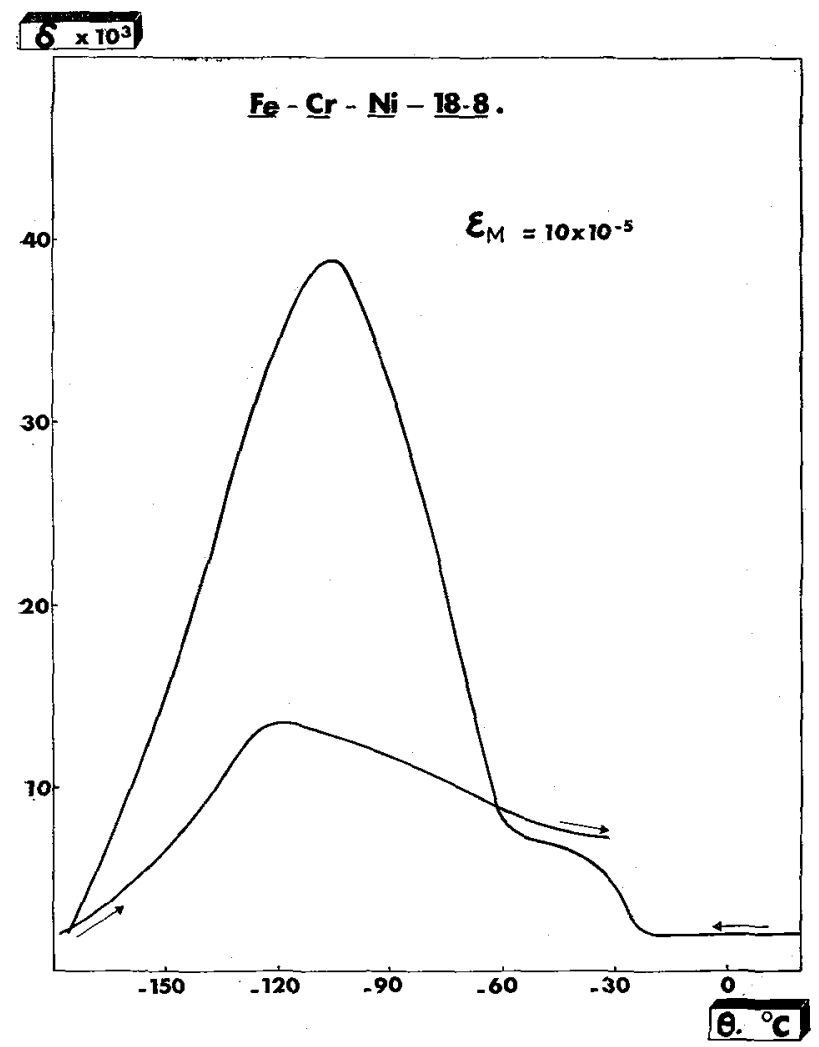

FIG. 9. -- Alliage Fer-Chrome-Nickel. Variation du frottement intérieur au cours du refroidissement et du réchauffage mesure effectuée à $\varepsilon=10^{-4}$. l'amplitude des oscillations entre $1,9 \times 10^{-5}$ et $7,3 \times 10^{-5}$.

D'autre part, il est probable que les décalages brutaux de la position d'équilibre peuvent être attribués au phénomène de burst comme les déformations brutales de la figure 1.

Pour l'alliage Co-Ni recuit, les résultats obtenus lors d'un chauffage jusqu'à $200^{\circ} \mathrm{C}$ suivi d'un refroidissement jusqu'à $-150^{\circ} \mathrm{C}$, sont portés sur la figure 8 . On constate l'existence de 2 maximums aigus, l'un au chauffage vers $180^{\circ} \mathrm{C}$, l'autre au refroidissement vers $-40^{\circ} \mathrm{C}$, correspondant respectivement à la

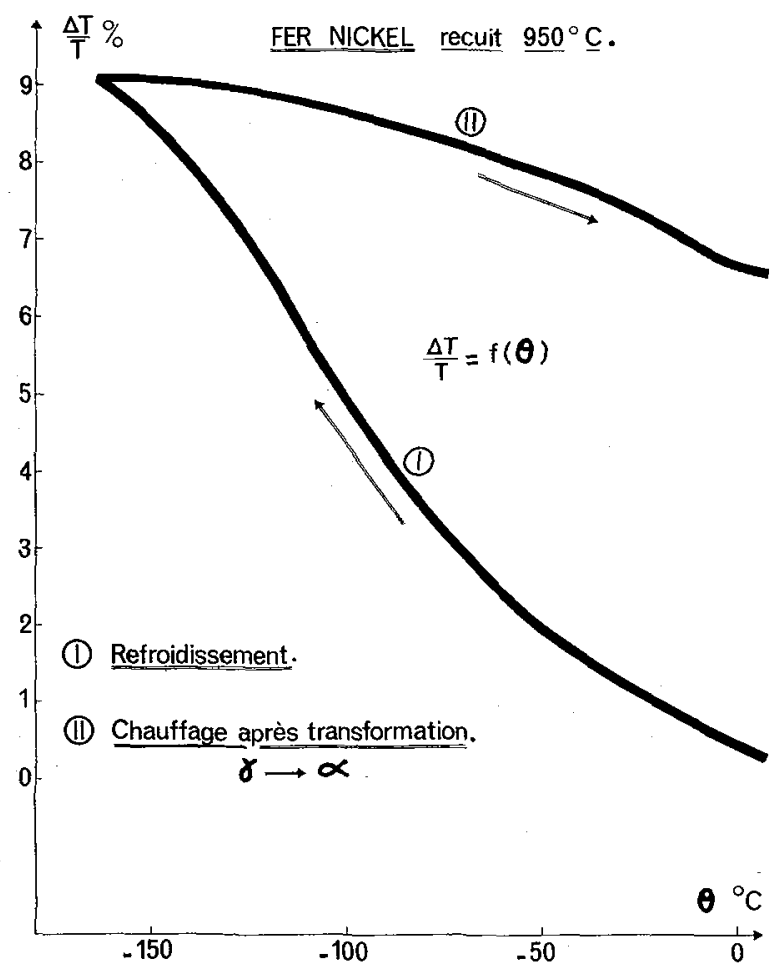

FIG. 10. - Alliage Fer-Nickel. Etude de la période d'oscillations au cours du refroidissement (I) et du réchauffage de l'alliage (II).

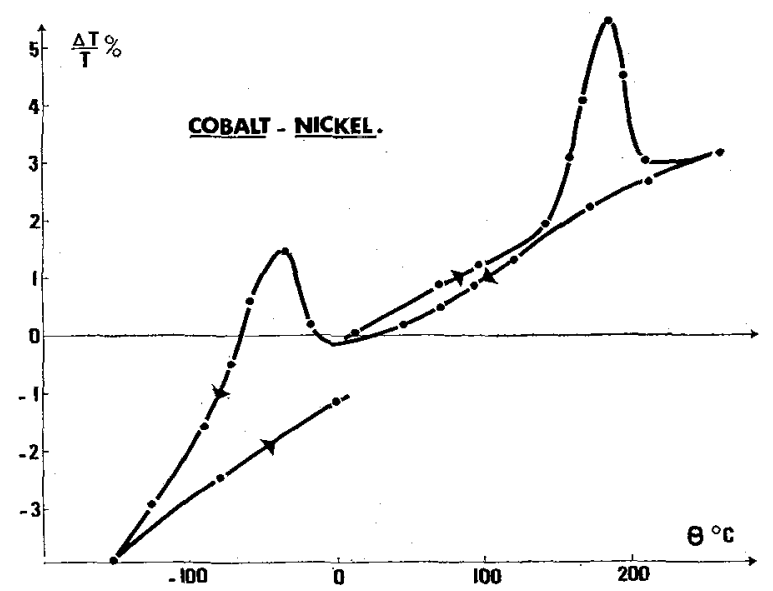

FIG, 11. - Alliage Cobalt-Nickel. Etude de la période d'oscillation au cours de la transformation h. c. $\rightarrow$ c. f. c. (chauffage) et c. f. c. $\rightarrow$ h. c. (refroidissement). 
transformation h. c. $\rightarrow$ c. f. c. et à la transformation inverse. L'amplitude des oscillations était de $2 \times 10^{-5}$.

Nous avons porté sur la figure 9 l'évolution du frottement intérieur pour l'alliage $\mathrm{Fe}-\mathrm{Cr}$-Ni mesuré à une amplitude de déformation maximale de $10 \times 10^{-5}$. On constate la présence de deux maximums, au refroidissement et au chauffage, dans le même domaine de température que les points d'inflexion des courbes de la figure 3, c'est-à-dire vers $-115^{\circ} \mathrm{C}$, température à laquelle la vitesse de transformation est maximale. On vérifie encore que la transformation c. f. c. $\rightarrow$ c. c. n'est pas totale au refroidissement, mais reprend plus faiblement lors du réchauffage à l'ambiante.

Nous avons donc bien vérifié pour les trois alliages étudiés que $\delta$ passe par un maximum dans les zones de température correspondant à des transformations. Pour les alliages $\mathrm{Co}-\mathrm{Ni}$ et $\mathrm{Fe}-\mathrm{Cr}-\mathrm{Ni}$, le maximum de $\delta$ a lieu pour la même température que celui de la vitesse de transformation.

III.3 VARIATIONS DE PÉRIODE. - Nous avons porté sur les figures 10 et 11 quelques résultats concernant les variations relatives de période pour les alliages $\mathrm{Fe}-\mathrm{Ni}$ et $\mathrm{Co}-\mathrm{Ni}$ en fonction de la température.

Pour l'alliage $\mathrm{Fe}-\mathrm{Ni}$ on remarque sur la figure 10 que la variation de période est plus importante lors de la transformation $\gamma \rightarrow \alpha$ au refroidissement que pour le réchauffage ultérieur.

Pour l'alliage Co-Ni recuit, on constate sur la figure 11 que l'anomalie de période présente 2 maximums très nets, le $1^{\mathrm{er}}$ au chauffage vers $180^{\circ} \mathrm{C}$, le second au refroidissement vers $-40^{\circ} \mathrm{C}$, c'est-à-dire aux mêmes températures que les maximums de frottement intérieur de la figure 8 .

IV. Discussion. - Nous avons tenté de relier les différents résultats expérimentaux obtenus en faisant l'hypothèse que la loi proportionnelle de microdéformation obtenue pour tout l'intervalle de température correspondant à une transformation demeurait valable localement, à un instant donné :

Nous posons donc (voir annexe) :

$$
\Delta \varepsilon_{p}=\frac{\mathrm{d} f(n)}{\mathrm{d} n} \cdot \Delta n \cdot \varepsilon
$$

où $\Delta \varepsilon_{p}$ est l'accroissement de déformation plastique pendant le temps $\Delta t$.

$\Delta n$ est la variation de concentration relative en martensite au cours du même intervalle de temps $\Delta t$.

$\mathrm{f}(n)$ est le coefficient de proportionnalité entre microdéformation plastique et déformation élastique.

$\varepsilon$ est la déformation élastique appliquée à l'instant $t$.

L'étude phénoménologique effectuée dans l'annexe permet de prévoir les résultats suivants :

1. Le frottement intérieur est indépendant de l'amplitude de la déformation appliquée.
2.: Le frottement intérieur est inversement proportionnel à la fréquence d'oscillation.

3. Le frottement intérieur est proportionnel à la vitesse de transformation $\mathrm{d} n / \mathrm{d} t$.

4. L'intégrale par rapport à la température de la courbe de frottement intérieur est une grandeur plus significative que le frottement intérieur lui-même. Cette intégrale est proportionnelle à la vitesse de variation de la température (eq. 7 de l'annexe) et peut être reliée à la microdéformation plastique obtenue lors des essais sous contrainte statique.

5. Lorsque la fonction $\mathrm{f}(n)$ est linéaire, l'intégrale $\int_{\theta_{1}}^{\theta_{2}} \delta(\theta) \mathrm{d} \theta$ est proportionnelle à la quantité de phase qui se transforme.

6. L'anomalie de période est proportionnelle au frottement intérieur.

Nous nous sommes attachés à la vérification de ces différents points, et nous pouvons faire les remarques suivantes :

1. Le frottement intérieur semble bien en première approximation indépendant de l'amplitude d'oscillation.

Pour l'alliage $\mathrm{Fe}-\mathrm{Ni}$, on n'a décelé aucune évolution pour des amplitudes de déformation allant de $2 \times 10^{-5}$ à $8 \times 10^{-5}$.

Dans le cas de l'alliage Co-Ni, la figure 12 fait apparaître une légère remontée du frottement intérieur aux basses amplitudes. L'augmentation qui apparait aux plus fortes amplitudes $\left(\varepsilon>4 \times 10^{-5}\right)$ est liée à la déformation plastique.

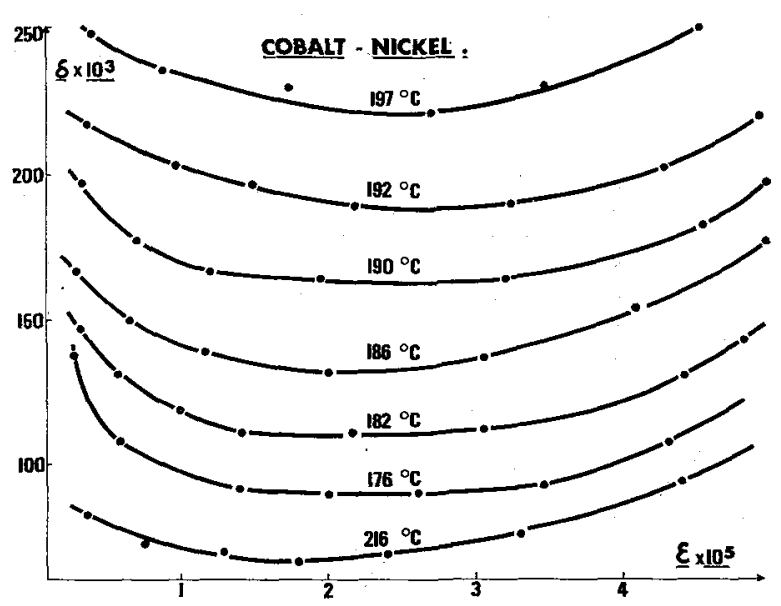

FIG. 12. - Alliage Cobalt-Nickel. Etude du frottement intérieur en fonction de l'amplitude de la sollicitation au cours de la transformation h. c. $\rightarrow$ c.f. c. (mesures effectuées en dynamique).

Pour l'alliage $\mathrm{Fe}-\mathrm{Cr}-\mathrm{Ni}$, des essais ont été effectués dans une gamme étendue d'amplitudes de déformation $\left(2 \times 10^{-7}<\varepsilon<2 \times 10^{-4}\right) \quad$ [23]. L'effet de l'amplitude paraît négligeable. 
2. L'influence de la fréquence n'a pu être mise en évidence de façon aussi claire. Cependant, des essais préliminaires sur l'alliage $\mathrm{Fe}-\mathrm{Cr}-\mathrm{Ni}$ effectués en haute fréquence $(1050 \mathrm{~Hz})$ ont montré que le frottement intérieur devenait très faible. Postnikov [20] a montré sur un alliage $\mathrm{Co}-\mathrm{Ni} 23 \%$ une telle influence de la fréquence.

3. La proportionnalité avec la vitesse de transformation n'a pas été mise en évidence dans cette étude. En revanche, elle a été démontrée en 1956 par Scheil et Muller [7] sur un alliage $\mathrm{Fe}-\mathrm{Ni}$ et par Delorme et al. [22] sur un alliage $\mathrm{Fe}-\mathrm{Cr}-\mathrm{Ni}$.

4. La relation [7] de l'annexe qui exprime la proportionnalité de l'intégrale $I$ à la vitesse de variation de la température a été convenablement vérifiée dans le domaine des vitesses utilisées

$$
\left(100^{\circ} \mathrm{C} / \mathrm{h}<\alpha<600^{\circ} \mathrm{C} / \mathrm{h}\right)
$$

malgré la mauvaise homogénéité de température aux grandes vitesses. Cette loi permet de penser que pour des vitesses infiniment lentes (état stationnaire) le frottement intérieur doit être réduit à son fond continu. Ce point a été mis en évidence expérimentalement par Selle dans des études sur le cobalt [21].

5. Les mesures d'anomalie de période sont en assez bon accord avec les prévisions. Dans l'alliage $\mathrm{Co}-\mathrm{Ni}$ où le frottement intérieur est très important on met en évidence un maximum d'anomalie de période. La figure 13 représente l'anomalie de période en fonction du frottement intérieur. Elle fait apparaître une proportionnalité correcte entre ces deux grandeurs.

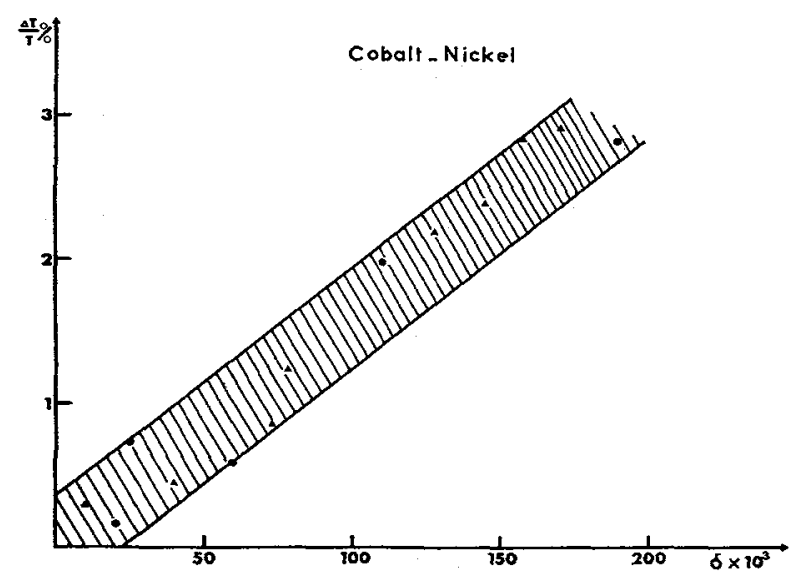

FIG. 13. - Alliage Cobalt-Nickel. Variation de l'anomalie de période en fonction du frottement intérieur :

$\Delta$ transformation h. c. $\rightarrow$ c.f.c. (chauffage);

- transformation c. f.c. $\rightarrow$ h. c. (refroidissement).

Il faut souligner la difficulté d'appréciation de l'anomalie de période car le fond continu n'est pas bien défini ; sa valeur évolue au cours de la transformation avec la température et la quantité de phase transformée. Dans les deux autres alliages les variations sont trop faibles pour être séparées clairement de la variation continue.

V. Interprétation. - Garber et Datsko [4] attribuent la dissipation d'énergie mécanique pendant la transformation à trois causes principales :

$$
\delta=\delta_{1}+\delta_{2}+\delta_{3} .
$$

Le premier terme $\delta_{1}$ serait le fond continu régulièrement observé dans les expériences de frottement intérieur et dont la cause est complexe. Cette contribution globale est d'autant plus faible que la température est basse, et nos conditions expérimentales sont de ce point de vue favorables.

Le terme $\delta_{2}$ serait une composante de frottement reliée au processus de transformation et localisée aux interfaces. On peut émettre l'hypothèse que cette composante est associée à un déplacement réversible des interfaces pendant la transformation. Cependant, il ne s'agit pas ici d'un pic de joint d'interphase tel que ceux qui ont été observés dans certains alliages d'aluminium [24], car la dissipation d'énergie est associée à la cinétique de transformation et s'annule lorsque la transformation est terminée. Il ne semble pas impossible de penser que le mécanisme de dissipation d'énergie est lié à l'interface en cours de propagation et aux dislocations qui le composent habituellement [25]. Il semble cependant très difficile d'évaluer cette contribution.

Le dernier terme $\delta_{3}$ serait dû aux pertes d'énergie associées à la microdéformation plastique. Les résultats que nous présentons et le parallèle entre frottetement intérieur et microdéformation permettent de penser que cette cause de dissipation est importante. On peut avancer deux hypothèses pour rendre compte de la microdéformation plastique [26].

- La déformation est associée au cisaillement qui accompagne la transformation martensitique.

- La déformation est associée au déplacement des dislocations nécessaire à l'accommodation des contraintes internes. Greenwood et Johnson [27] ont déjà étudié cet aspect; de telles dislocations ont pu être observées par microscopie électronique [28].

Une étude quantitative est nécessaire pour préciser la part qui revient à chacun de ces processus.

VI. Conclusion. - Cette étude avait pour but de mettre en évidence les effets de microdéformation sous faible charge qui accompagnent les transformations martensitiques. Il a été possible d'établir un lien correct entre les essais sous contrainte statique et les essais sous contrainte cyclique et d'estimer qualitativement l'influence des divers paramètres.

Il apparait raisonnable, pour obtenir un meilleur accord et expliquer le frottement intérieur d'étudier en détail les divers processus physiques possibles ce qui devrait permettre de préciser l'interaction martensite-matrice au cours même de la transformation. 


\section{Bibliographie}

[1] WASSERMANN (G.), Arch. Eisenhüttenwesen, 1937, 321.

[2] Sauveur (A.), Iron Age, 1924, 113, 581.

[3] Kot (R.), Krause (G.), Weiss (V.), “ Transformation plasticity of titanium ), Communication en 1968 au Fall Meeting of AIME,

[4] Garber (R. I.), DatSko (O. I.), Fiz. metal. metalloved., $1965,20,442$.

[5] Bungardt (K.), Preisendanz (H.), Brandis (H.), DEW Technische Berichte, 1962, 2, 59.

[6] SCheil (E.), THIELE (W.), Arch. Eisenhüttenwesen, 1938, 12, 103.

[7] ScheIL (E.), Muller (J.), Arch. Eisenhüttenwesen, $1956,27,801$.

[8] Postnikov (V. S.), Fiz. metal. metalloved, 1957, 4, 344.

[9] Bungardt (K.), Preisendanz (H.), Brandis (H.), Arch. Eisenhüttenwesen, 1961, 32, 113.

[10] Koster (W.), Zeit. Metallkunde, 1948, 39, 1.

[11] Portevin (A.), Cabarat, C. R. Acad. Sci. Paris, $1956,243,1085$.

[12] Ye Ivanov (V.), Shapovol (B. I.), Amonenko (V. M.), Fiz. metal. metalloved, 1961, 11, 52.

[13] Jones, Pumphrey, $J$ ISI, 1949, 121.

[14] Bibring (H.), Lenoir (G.), Sebilleau (F.), Rev. metallurgie, 1959, LVI, 279.
[15] Coulomb (P.), Mem. Sc. Rev. Met., 1968, LXV, 149.

[16] СесH (R. E.), Hollomon (J. H.), Trans. AIME, 1953 685.

[17] Imai (Y.), Izumiyama (M.), Sasaki (K.), Sc. Rep. Res. Inst. Tokohu Univ. 1966, A 18, 39.

[18] ReEd (R. P.), Acta Met., 1962, 10, 865

[19] Postnikov, Belko (V. N.), Sharshakov (I. M.), Fiz. Metallov Metalloved, 1968, 26, 1051

[20] Postnikov, Belko (V. N.), Darinski (B. M.), SharshaKov (I. M.), Fiz, Metallov Metalloved $1969,27,141$.

[21] Selle (J. E.), Cobait, 1969, 45, 186.

[22] Delorme (J. F.), Robin (M.), Gobin (P.), Mem. Sci. Rev. Met., 1969, LXVI, 125.

[23] Bays, Laboratoire de Génie Atomique, Ecole Polytechnique Fédérale de Lausanne, Communication privée.

[24] Schoeck (G.), Bisogni (E.), Phys. Stat. Sol., 1969, $32,31$.

[25] Frank (F. C.), Acta Met., 1953, 1, 15.

[26] Magee (C. L.), Paxton (H. W.), Trans. AIME, 1968, 242,1741 .

[27] Greenwood (G. W.), Johnson (R. H.), Proceedings of the Royal Society of London, 1965, 283, 403 .

[28] Dash (S.), Brown (N.), Acta Met., 1966, 14, 595. 


\section{ANNEXE \\ ÉTUDE COMPARÉE DU FROTTEMENT INTÉRIEUR DE LA PÉRIODE D'OSCILLATION ET DE LA MICRODÉFORMATION}

On peut tenter d'établir un lien entre nos différents essais en considérant les résultats des essais de microdéformation. On a montré expérimentalement que pour un taux de transformation donné la déformation plastique $\varepsilon_{p}$ est proportionnelle à la déformation élastique $\varepsilon_{i}$ (c'est-à-dire à la contrainte appliquée $\left.\sigma=G \cdot \varepsilon_{i}\right)$

$$
\varepsilon_{p}=p \cdot \varepsilon_{i} .
$$

Il semble d'après les résultats obtenus sur les alliages $\mathrm{Co}-\mathrm{Ni}$ et $\mathrm{Fe}-\mathrm{Cr}-\mathrm{Ni}$ (Fig. 5 et 6 ) que cette proportionnalité demeure lorsque les quantités relatives transformées varient, tant que la contrainte statique appliquée $\sigma$ n'est pas suffisante pour induire la transformation. Dans l'équation (1) le coefficient de proportionnalité $p$ est alors une fonction croissante de la concentration relative en phase transformée $n$.

$$
\varepsilon_{p}=\mathrm{f}(n) \cdot \varepsilon_{i}
$$

où $f(n)$ satisfait à $f(0)=0$.

Tant que la concentration $n$ n'est pas trop importante on peut considérer que chaque élément de volume qui se transforme apparait dans la matrice sans trop interagir avec les éléments voisins $\left(^{*}\right)$ : la fonction $\mathrm{f}(n)$ doit être alors linéaire

$$
\mathrm{f}(n)=A . n \text {. }
$$

L'hypothèse de notre calcul consiste à admettre que l'expression (2) valable pour une transformation donnée reste valable localement à tout instant. En différentiant l'équation (2) on peut écrire l'accroissement de déformation plastique $\Delta \varepsilon_{p}$ correspondant à une déformation élastique $\varepsilon$ pendant un temps $\Delta t$ où se transforme la quantité $\Delta n$

$$
\begin{aligned}
& \Delta \varepsilon_{p}=\frac{\mathrm{df}(n)}{\mathrm{d} n} \Delta n \cdot \varepsilon \\
& \Delta \varepsilon_{p}=\frac{\mathrm{df}(n)}{\mathrm{d} n} \cdot \frac{\mathrm{d} n}{\mathrm{~d} t} \cdot \Delta t \cdot \varepsilon
\end{aligned}
$$

et sous forme dérivée

$$
\dot{\varepsilon}_{p}=\frac{\mathrm{df}(n)}{\mathrm{d} n} \cdot \frac{\mathrm{d} n}{\mathrm{~d} t} \cdot \varepsilon
$$

(*) On pent situer la concentration critique au voisinage de $15 \%$. Dans ce cas' en effet un volume sphérique de rayon $r$ se transforme dans une matrice sphérique de rayon $2 r$.
A. Essai de frottement intérieur. - La vitesse de déformation plastique $\varepsilon_{p}$ donnée par l'équation (4) permet de calculer le frottement intérieur $\delta$, lorsque la contrainte appliquée $\sigma$ est sinusoïdale $\varepsilon=\varepsilon_{0} \sin \omega t$ $\delta=\frac{1}{G \varepsilon_{0}^{2}} \oint \sigma \dot{\varepsilon}_{p} \cdot \mathrm{d} t \delta=\oint \frac{\mathrm{d} f(n)}{\mathrm{d} n} \cdot \frac{\mathrm{d} n}{\mathrm{~d} t} \cdot \sin ^{2} \omega t \cdot \mathrm{d} t$.

On suppose que le phénomène physique (transformation martensitique) est continu et que la période du cycle est suffisamment courte pour que le terme $\frac{\mathrm{d} f(n)}{\mathrm{d} n} \cdot \frac{\mathrm{d} n}{\mathrm{~d} t}$ soit constant pendant le cycle, on obtient:

$$
\delta=\frac{\pi}{\omega} \cdot \frac{\mathrm{df}(n)}{\mathrm{d} n} \cdot \frac{\mathrm{d} n}{\mathrm{~d} t} .
$$

- Le frottement intérieur est indépendant de l'amplitude de sollicitation.

- Il est inversement proportionnel à la fréquence.

- Il dépend de la vitesse de transformation $\mathrm{d} n / \mathrm{d} t$.

- Si la fonction $\mathrm{f}(n)$ est linéaire, le frottement intérieur est proportionnel à la vitesse de transformation $\delta=(\pi / \omega) \cdot A \cdot(\mathrm{d} n / \mathrm{d} t)$

B. Relation entre microdéformation et frottement intérieur. - L'équation (5) traduit la relation locale, c'est-à-dire pendant un temps égal à la période $T$ d'un cycle de soliicitation, entre $\delta$ et $(\mathrm{df} / \mathrm{d} n) .(\mathrm{d} n / \mathrm{d} t)$.

Dans le cas particulier où $\varepsilon$ est considéré comme constant et égal à $\varepsilon_{i}$ pendant le temps $T$, on peut utiliser cette relation, ce qui, en tenant compte de l'équation (4), permet d'écrire :

$$
\dot{\varepsilon}_{p}=\varepsilon_{i} \frac{\omega}{\pi} \delta
$$

Lorsque le phénomène étudié comme c'est le cas ici, dépend de la température, il est possible d'obtenir l'expression intégrée de l'équation (6) qui permet de relier la déformation plastique totale $\varepsilon_{p}$ à la courbe de frottement intérieur. Soit $\alpha$ la vitesse de variation de la température $\alpha=\mathrm{d} \theta / \mathrm{d} t$

$$
\begin{aligned}
\varepsilon_{p}=\int_{0}^{t} \dot{\varepsilon}_{p} \cdot \mathrm{d} t=\int_{\theta_{1}}^{\theta_{2}} \dot{\varepsilon}_{p} & \cdot \frac{1}{\alpha} \cdot \mathrm{d} \theta= \\
& =\frac{\omega}{\pi} \cdot \varepsilon_{i} \cdot \int_{\theta_{1}}^{\theta_{2}} \delta(\theta) \cdot \frac{1}{\alpha} \cdot \mathrm{d} \theta
\end{aligned}
$$


Lorsque la température varie linéairement avec le temps, il vient

$$
I=\int_{\theta_{1}}^{\theta_{2}} \delta(\theta) \cdot \mathrm{d} \theta=\pi \cdot \frac{\alpha}{\omega} \cdot \frac{\varepsilon_{p}}{\varepsilon_{i}} .
$$

Cette expression fait apparaître que la grandeur importante n'est pas l'amplitude du frottement intérieur, mais son intégrale. Celle-ci est proportionnelle à la vitesse de variation de la température.

$\delta$ représente à un facteur de proportionnalité près la dérivée de la courbe $\varepsilon_{p}=\mathrm{f}(\theta)$.

Lorsque la fonction $f(n)$ est linéaire on a l'expression

$$
I=\int_{\theta_{1}}^{\theta_{2}} \delta(\theta) \cdot \mathrm{d} \theta=K n
$$

L'intégrale est alors proportionnelle à la quantité de phase transformée.

C. Etude de la période d'oscillation. - On sait que la période $T$ des oscillations est liée au module apparent $G_{a}$ du matériau, que l'on peut définir par le rapport de la contrainte à la déformation totale obtenue sur un quart de cycle :

$$
G_{a}=\frac{\sigma_{0}}{\varepsilon_{i}+\varepsilon_{p c}}
$$

où

$$
\varepsilon_{p c}=\int_{0}^{T / 4} \frac{\mathrm{df}(n)}{\mathrm{d} n} \cdot \frac{1}{G} \cdot \frac{\mathrm{d} n}{\mathrm{~d} t} \sigma_{0} \sin \omega t \cdot \mathrm{d} t
$$

On peut intégrer en supposant $(\mathrm{d} n / \mathrm{d} t)$ et $(\mathrm{df}(n) / \mathrm{d} n)$ constants, d'où

$$
\varepsilon_{p c}=\frac{\mathrm{df}(n)}{\mathrm{d} n} \cdot \frac{\mathrm{d} n}{\mathrm{~d} t} \cdot \frac{\sigma_{0}}{G} \cdot \frac{1}{\omega} .
$$

En combinant les équations (5), (9) et (10), on obtient :

$$
G_{a}=\frac{\sigma_{0}}{\varepsilon_{i}\left(1+\frac{\delta}{\pi}\right)}=\frac{G}{1+\frac{\delta}{\pi}} .
$$

On définit alors l'anomalie de module

$$
\frac{\Delta G}{G}=\frac{G_{a}-G}{G} \simeq-\frac{\delta}{\pi}
$$

et l'anomalie de période :

$$
\frac{\Delta T}{T}=-\frac{1}{2} \frac{\Delta G}{G}=\frac{\delta}{2 \pi} .
$$

L'anomalie de période varie proportionnellement à $\delta$.

Remarque. - Les calculs ont été menés avec les hypothèses suivantes :

$a$. On a supposé la continuité du phénomène physique ce qui a permis d'effectuer normalement les intégrations.

$b$. On a admis que l'effet d'anélasticité reste le même que l'on travaille sous contrainte statique ou sous contrainte sinusoïdale.

Il est raisonnable de penser que ces hypothèses ne peuvent servir à un calcul quantitatif réel, mais permettent seulement de déterminer qualitativement l'influence des différents paramètres. Il est certain en effet que la transformation martensitique est essentiellement discontinue, sans que l'on puisse d'ailleurs en préciser la cinétique, si ce n'est par des mesures à réponse très rapide.

D'autre part il est probable que le phénomène anélastique n'est pas exactement le même lorsqu'il se produit sous contrainte statique ou sous contrainte cyclique. 